the sequencing centres and their collaborators are seeking ways to provide added value to the unpublished sequence data.

At a meeting of the malaria genome project in June at the Wellcome Trust Genome Campus in Hinxton, UK, the sequencing centres agreed that a first-pass annotation would be added to their websites, including posting the results of blast hits for all the open reading frames. Participants in the malaria genome project have agreed on the development of the PlasmoDB database (http://plasmodiumdb.cis.upenn.edu), a web-accessible relational database facilitating the analysis of various genome-related data. With support from a grant from the Burroughs Wellcome fund, this database is being developed by David Roos and his colleagues at the University of Pennsylvania and by Ross Coppell at Monash University.

Contrary to your News report, the PlasmoDB team is not supported for, or engaged in, any effort to annotate the genome. Discussion is under way, however, to determine the extent of automated annotation that will be provided through PlasmoDB for unfinished sequence available from the sequencing centres.

WHO's programme for research and training in tropical diseases (see below) is to provide support for a 'help desk', in addition to the current distribution of data through a CD-ROM version of Malaria Information Resource, to help investigators who lack the computational resources to access and analyse the data.

Michael Gottlieb ${ }^{\star}$, Victoria McGovern $\dagger$, Patricia Goodwin $\neq$, Stephen Hoffman§, Ayo Oduolall

${ }^{*}$ National Institute of Allergy and Infectious Diseases, 6700-B Rockledge Drive, Bethesda, Maryland 20892, USA

$\dagger$ Burroughs Wellcome Fund, 21 T. W. Alexander Drive, Research Triangle Park, North Carolina 27709, USA

$\ddagger$ Wellcome Trust, 183 Euston Road, London NW1 2BE, UK

$\$ N$ Naval Medical Research Center, 503 Robert Grant Avenue, Silver Spring, Maryland 20910-7500,USA IIUNDP/World Bank/WHO Special Programme for Research and Training in Tropical Diseases (TDR), 1211 Geneva 27, Switzerland

\section{... when public-interest science needs solidarity}

Sir- As a member of the small community of people working on malarial parasites, I am a constant user of sequence databases from the consortia at Sanger Centre, TIGR and Stanford (Nature 405; $601-602 ; 2000$ ). I am deeply grateful to these centres, which release all the data freely on the Internet, even in raw form, as soon as they are available.
According to the data-release policy of these three consortia, "these data will assist colleagues in their research, particularly in the search for genes and the studies of the genes' biological function" and "provide investigators with information that may jump-start biological experimentation". New perspectives are being added to virtually all the ongoing research projects and many more projects will start, as well as other 'brute force' approaches such as roteomix and SAGE (serial analysis of gene expression). All these data together will be integrated by bioinformaticists into more sophisticated and complete databases.

I sympathize with those who say that a period of "non-hypothesis science" has started, but I don't agree with them. Good scientists will go on making good hypotheses, and the vastness of available data will only eliminate time-consuming and painstaking benchwork. As things stand, much laboratory work contributes to the efforts of the sequencers by providing clues for annotation and function. In the near future, many other hints will help to clarify the fine structure of the sequenced regions, for example when introns and exons are present that are too small to be revealed by the currently used algorithms, or when regulatory regions need to be identified.

For these reasons, I found the quarrel between the sequencing consortia and laboratory researchers reported in your News article very worrying. It would of course be wrong for a tribe of 'annotators' to develop, exploiting raw data only for their own publications instead of helping the sequencers. But, again, the release policy states that any investigator should ask permission of the consortia to publish data based on the content of the databases, and referees should easily be able to distinguish between a genuine contribution and data piracy. In this regard, the PlasmoDB database of Roos and colleagues represents an important tool for all of us and cannot be described simply as an "Internet portal" or connected with the concept of "piracy".

Scientific careers are largely based on the amount and quality of publications, but it would be impossible for every new chromosome of every organism to be published in the highest-impact journals such as Nature and Science. Perhaps an online journal or supplement dedicated to sequencing and annotation work should be launched.

The most serious aspect of this situation is, however, not mentioned in the malaria article but is well put in another News report on the same pages: "Drive for more genomes threatens mouse sequence" (Nature 405; 602-603; 2000).

Craig Venter of Celera Genomics is quoted as stating that the mouse genome is almost correctly assembled, directed by the blueprint of the human genome - that is, by fundamental work done by public enterprise and non-profit consortia. In spite of this, Venter says Celera's mouse genome will be restricted to subscribers.

\section{Tomasino Pace}

Laboratorio di Biologia Cellulare, Istituto Superiore di Sanità, Viale Regina Elena 299, 00161 Rome, Italy

\section{The search continues for Kármán's St Christopher}

Sir - It is nice to see fourteenth-century Bolognese frescoes getting a bit of welldeserved publicity in the Science in Culture article "St Christopher and the vortex"1. However, if Theodore von Kármán was inspired by the beautiful San Domenico fresco of St Christopher to conceive of his 'vortex street', his powers as a psychic would be at least as remarkable as his achievements in the physical sciences.

True, the fresco in the church of San Domenico was painted during the late fourteenth century (to a formula much used by Tomaso da Modena and his associates $^{2}$ ) and Kármán was born in 1881. But it had been hidden since $1728-31$ behind a staircase built up to an organ loft. It was only revealed during repairs in 1979.

So what was the painting that Kármán reported had inspired him? Mizota and his partners are surely correct in seeing fourteenth-century painting style in Kármán's water-flow representation, but today remarkably few examples of St Christopher survive in Bologna itself: the grandest, in San Petronio, by Giovanni da Modena (circa 1410-20), has been cut off at the ankles. Tomaso da Modena painted a similar image in San Francesco, Treviso, around 1350. A pupil of his painted another in the next chapel; similar accumulations probably occurred in churches in Bologna, but were lost during the Counter-Reformation.

The representation of St Christopher that Kármán is most likely to have seen is the triptych by Jacopo di Paolo from the 1390 s that has long been shown in various rooms of the outer cloisters of San Stefano ${ }^{3}$ - but this comes nowhere near the San Domenico fresco in the clarity with which it shows water flowing.

We might deduce from this that von Kármán was sufficiently sensitive to painting style to have read the appropriate patterns into what he saw, from his own knowledge of both art and water, in his moment of inspiration.

\section{Robert Gibbs}

Department of History of Art, University of Glasgow, $3 l$ Albany Mansions, 351 Renfrew Street, Glasgow G3 6UW, UK

\footnotetext{
1. Mizota, T. et al. Nature 404, 226 (2000).

2. D'Amico, R. Strenna Storica Bolognese 33, 95-113 (1983).

3. Arcangeli, F. Pittura Bolognese del '300 (Grafis, Bologna, 1978).
} 